\title{
Tipping the balance towards resolution
}

Tristetraprolin (TTP), a key negative regulator of proinflammatory cytokine production, could be a novel therapeutic target for patients with rheumatoid arthritis (RA), new research suggests. By manipulating the phosphorylation status of TTP, Andy Clark's group at the University of Birmingham, UK, found that they could halt the development of inflammatory arthritis in the $\mathrm{K} / \mathrm{B} \times \mathrm{N}$ serum-transfer mouse model. "The results surpassed our expectations, the mice expressing a mutant form of TTP being completely protected against symptoms of arthritis," enthuses Clark. "We were so surprised that we repeated the experiment three times using different time frames or sources of arthritogenic serum, and observed exactly the same result," he explains.

As part of the mitogen-activated protein kinase (MAPK) p38 signalling pathway, TTP negatively regulates the expression of genes

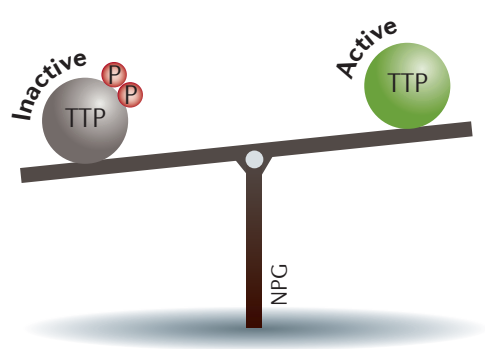

encoding proinflammatory proteins by destabilizing their mRNAs. The phosphorylated, inactive form of TTP is dephosphorylated (and thereby activated) by serine/ threonine-protein phosphatase $2 \mathrm{~A}$ (PP2A). Consequently, increased activation of TTP reduces the production of proinflammatory cytokines.

Clark and colleagues found high levels of TTP in the synovium of patients with RA, compared with levels in non-inflamed synovium from patients undergoing arthroscopy. TTP was strongly expressed in cells in which the MAPK p38 signalling cascade was active: vascular endothelial cells, macrophages and a subset of fibroblasts. "We want to investigate the phosphorylation status of TTP in the rheumatoid synovium, testing the hypothesis that the TTP protein which accumulates at this site is phosphorylated and inactive," states Clark.

The researchers used the PP2A-activating agents COG1410 (an apolipoprotein E mimetic) and $\mathrm{AAL}_{(\mathrm{S})}$ (a derivative of fingolimod) to promote dephosphorylation of TTP. Both agents reduced arthritis progression and severity, as well as protecting against ankle swelling and bone erosion, in mice with wild-type TTP. In vitro, COG1410 reduced the expression of TNF in bone marrow-derived macrophages from mice with wild-type TTP, but did not reduce the (already low) expression of TNF in cells from mice with active mutant (non-phosphorylatable) TTP. COG1410 also reduced TNF-induced IL-8 expression in fibroblast-like synoviocytes from patients with RA, and decreased lipopolysaccharideinduced expression of TNF in human monocyte-derived macrophages.

Both COG1410 and $\mathrm{AAL}_{(\mathrm{S})}$ exert their anti-inflammatory effects by tipping the balance towards dephosphorylation of TTP, leading the researchers to suggest that targeting TTP could represent a new approach to treating inflammatory diseases. "The MAPK p38 pathway was considered a very promising drug target until the conclusions of several clinical trials in RA and other chronic inflammatory diseases showed that the therapeutic effects of potent and specific p 38 inhibitors were disappointingly transient," explains Clark. "Nonetheless, it might still prove possible to achieve therapeutic effects by targeting this pathway using different approaches."

Joanna Collison

ORIGINAL ARTICLE Ross, E. A. et al. Treatment of inflammatory arthritis via targeting of tristetraprolin, a master regulator of pro-inflammatory gene expression. Ann. Rheum. Dis. http://dx.doi.org/ 10.1136/annrheumdis-2016-209424 (2016) 